Archives

$11 \mid 1993$

Histoire intellectuelle // Réflexions collectives sur I'histoire sociale

\title{
La Nouvelle-Calédonie, 1853-1920. Naissance d'une société coloniale
}

Isabelle Merle

\section{(2) OpenEdition \\ Journals}

Édition électronique

URL : http://journals.openedition.org/ccrh/2762

DOI : $10.4000 /$ ccrh. 2762

ISSN : $1760-7906$

Éditeur

Centre de recherches historiques - EHESS

Édition imprimée

Date de publication : 10 octobre 1993

ISSN : 0990-9141

Référence électronique

Isabelle Merle, «La Nouvelle-Calédonie, 1853-1920. Naissance d'une société coloniale », Les Cahiers du Centre de Recherches Historiques [En ligne], 11 | 1993, mis en ligne le 05 mars 2009, consulté le 23 avril 2019. URL : http://journals.openedition.org/ccrh/2762 ; DOI : 10.4000/ccrh.2762

Ce document a été généré automatiquement le 23 avril 2019

Article L.111-1 du Code de la propriété intellectuelle. 


\title{
La Nouvelle-Calédonie, 1853-1920. Naissance d'une société coloniale
}

\author{
Isabelle Merle
}

\section{NOTE DE L'AUTEUR}

Thèse de doctorat préparée sous la direction de Denys Lombard et soutenue en mars 1993.

\section{Enjeux d'histoire et de mémoire}

1 «Les événements, pour reprendre la formule consacrée, ont explosé en NouvelleCalédonie, en 1984, il y a maintenant près de dix ans. Les Français, à l'occasion, découvrent une île lointaine $\mathrm{du}$ Pacifique, un peuple Kanak, une communauté « caldoche » mais aussi une histoire jusqu'alors ignorée. En demandant l'indépendance, les Mélanésiens ne se contentent pas de dénoncer les inégalités du moment mais remettent radicalement en cause 150 ans de présence française. Ils révèlent, pour la première fois, un passé longtemps tu; passé réinvesti tel un enjeu essentiel de connaissance, de reconnaissance et d'identité. Aux revendications portant sur leur culture, leurs origines et leur monde, s'ajoute le rappel des injustices subies. Les spoliations foncières, les souvenirs de la révolte de 1878, les rigueurs du code de l'indigénat, la violence des colons, les travaux obligatoires, le fait colonial en général sont, au fond, les arguments forts d'un discours qui exige réparation.

Brutalement renvoyée à son passé, la France des années 1980 n'a pas toujours les moyens de comprendre. Si elle méconnaît les Kanaks, elle méconnaît aussi les «Blancs» et les autres communautés de l'île, produits d'une histoire oubliée. A travers le «dossier calédonien ", ce sont les scènes d'un autre siècle qui reviennent en mémoire : le bagne, la Commune, mais aussi l'épopée coloniale, la conquête, les valeureux colons, les espoirs et les déboires de la colonisation. Emergeant dans toute sa complexité, la Nouvelle- 
Calédonie force le souvenir; une île qui fut avec l'Algérie, une véritable colonie de peuplement et qui pose, aujourd'hui, des problèmes que l'on croyait définitivement résolus.

Pour l'historien, la Nouvelle-Calédonie offre un champ d'étude exceptionnel. Cet archipel, situé à l'exact antipode de la métropole, à $18000 \mathrm{~km}$ de distance, fait partie de ces « confettis d'empire » que l'historiographie française a tendance à ignorer. Trop petite, trop lointaine, trop marginale, cette île, comme d'ailleurs l'ensemble des territoires du Pacifique, échappe à l'évidence au renouveau que connaissent, à l'heure actuelle, les études coloniales, davantage tournées vers les « grandes colonies » : le Maghreb, l'Afrique Noire ou l'Indochine.

4 La Nouvelle-Calédonie pourtant, dès qu'on s'y intéresse, révèle une histoire étonnante qui, aussi paradoxal que cela puisse paraître, s'inscrit au coeur de "grandes questions ». Fondée sur un véritable projet idéologique de peuplement, cette colonie est le siège d'une expérience sans précédent dans le contexte de l'Empire français; expérience qui prétendait construire une société «nouvelle» mêlant en son sein condamnés et "honnêtes» migrants. Soumise à une double logique d'émigration contrainte et volontaire, la Nouvelle-Calédonie coloniale, calquée sur le modèle de "l'Australie blanche", est pensée tout d'abord comme un réceptacle privilégié pour les «classes dangereuses et laborieuses » d'une France hantée par ses peurs sociales. Les intérêts évoluant, l'île incarne, à la fin du siècle, une fonction plus positive, celle "d'une petite France Australe ", symbole du rayonnement d'un peuple et de la puissance d'une nation. La terre est au coeur de tous les enjeux puisqu'il s'agit d'offrir ce bien si précieux aux bagnards et aux migrants. Ainsi espère-t-on fonder aux antipodes, une petite paysannerie laborieuse, amendée ou satisfaite; un "nouveau monde» réparateur construit sur l'image des campagnes françaises idéalisées. Ce projet de peuplement, porteur de hautes ambitions, donne naissance à une société «blanche » disparate et surprenante ; société qui s'installe face à un monde mélanésien soumis à une politique indigène particulièrement sévère. Alors que partout ailleurs dans l'Empire, la France renonce à appliquer le cantonnement des autochtones, les Kanaks sont, quant à eux, relégués dans de véritables réserves, à l'instar des Indiens d'Amérique ou des Aborigènes d'Australie.

5 Au-delà des originalités propres au passé calédonien, ce pays présente à la fin des années 1980 une situation unique dans la mesure où l'histoire coloniale est là, encore en acte. Les «Caldoches » ou Kanaks s'affrontent sur le terrain même de leurs pères et grand-pères. Les " événements ", au fond, jouent et rejouent les scènes d'un drame ancien, réactivant une mémoire qui, seule, permet de comprendre le sens des paroles et des actes, des violences et des réconciliations. Cette mémoire vivante, enjeu central des questions actuelles, offre une opportunité qu'il fallait saisir rapidement: le recueil in situ d'une histoire orale témoignant d'un monde colonial passé et d'acteurs qui, n'écrivant pas, ont laissé très peu de traces derrière eux. Ainsi pouvait-on espérer atteindre des réalités ignorées, touchant aux catégories du vécu et aux mentalités.

\section{Problématique et méthode}

6 Le travail présenté ici, n'avait pas la prétention d'écrire une «histoire totale » de la colonisation française en Nouvelle-Calédonie. Ni l'état des recherches ${ }^{1}$, ni le contexte d'enquête ${ }^{2}$, en effet, ne le permettaient. 
7 Interpellé par la complexité de politiques coloniales multiformes et l'extrême hétérogénéité de la société «blanche" qui en résulte, nous avons fait le choix de concentrer notre attention sur la genèse du peuplement «blanc» en articulant précisément l'analyse autour des systèmes en jeu (théories et logiques coloniales) et des acteurs historiques eux-mêmes (itinéraires, trajectoires et reconstitution de nouvelles identités).

8 L'une des idées essentielles consistait à dépasser les clivages existant entre une histoire proprement «impériale» (macro) située au niveau des politiques étatiques et de leurs relais locaux (Gouverneurs et administrateurs) et une histoire « island-oriented» (micro) limitée aux seules frontières de l'île et focalisée sur les destinées de groupes restreints ${ }^{3}$. Nous voulions de ce fait, mettre en valeur une dynamique globale jouant sur plusieurs niveaux, (État, colonie et acteur) afin de rendre compte des processus déterminants en oeuvre dans la constitution de la société coloniale de Nouvelle-Calédonie. En remontant aux origines - des découvertes à la conquête, de la conquête à l'idée de peuplement nous tenions à poser, au préalable, les racines d'un développement colonial ; racines qui s'inscrivent à la fois dans l'histoire particulière d'une expansion dans le Pacifique aux buts multiples et souvent incertains et dans une problématique proprement française, touchant à la criminalité, à la misère et enfin à la question de l'émigration. Une fois ce contexte précisément analysé, il s'agissait de réduire la focale "au théâtre de l'action », l'île elle-même, progressivement bouleversée par la mise en oeuvre du projet colonial. Le pays mélanésien initial, les révoltes kanaks, la constitution des réserves, l'implantation du bagne, les développements chaotiques des colonisations dites " pénales » ou « libres », les écarts entre les principes et les réalités dessinent les logiques et les dérives d'un processus qui se voulait pourtant pensé et volontariste. Se précisent alors peu à peu les fondements d'un ordre colonial, la hiérarchisation d'une société naissante, la constitution d'un nouvel espace calédonien rigoureusement cloisonné.

Pour appréhender le processus dans toute sa finesse, nous avons eu recours à l'analyse intensive de réalités micro-sociales. Choisissant deux zones représentatives d'implantation pénale ou libre, il s'agissait de retracer la genèse de villages coloniaux à travers la reconstitution fidèle de la trajectoire des familles fondatrices. Usant d'une technique proche de la micro-histoire, nous avons utilisé toutes les sources disponibles relatives à ces lieux, sources écrites (État-civil ${ }^{4}$, registres de l'Administration pénitentiaire, courriers municipaux, lettres des missionnaires, comptes rendus du Conseil Général ou Privé etc.) mais aussi sources orales, à savoir entretiens systématiques avec les descendants. L'enjeu était à la fois de décrypter les réalités objectives d'une "histoire coloniale vue d'en bas » et d'analyser les logiques constitutives des représentations et des liens sociaux pour déboucher in fine sur la recomposition des identités.

Le schéma qui vient d'être présenté, supposait à l'évidence des choix qu'il convient d'expliciter. Les Kanaks, s'ils ne sont pas au centre de l'étude, interviennent à deux niveaux essentiels : d'une part à travers le système institutionnel qu'ils subissent (mise en réserve et interdictions de déplacements); système qui influe considérablement sur la structuration globale de la société coloniale et sur les rapports entre communautés, et d'autre part à travers les représentations dont ils sont l'objet ; représentations qui jouent un rôle central dans la constitution des identités coloniales. Le monde colonial, par ailleurs, ne pouvait être étudié dans toute sa diversité. Guidé par les dynamiques propres au projet initial, nous avons focalisé l'attention sur les deux branches principales de peuplement : la transportation des condamnés et l'émigration libre, « assistée » par l'État. 
Cette dernière, qui représente l'essentiel de la colonisation « volontaire » en NouvelleCalédonie, s'inscrit dans les programmes mis en place par le ministère des Colonies pour stimuler les départs; programmes qui offrent gratuitement le voyage, la concession de terre, les aides à l'installation en échange d'un certains nombres d'obligations (site d'implantation et surfaces imposés, contrôle sur l'état des cultures) ${ }^{5}$.

11 Enfin, la construction d'un monde rural constituant l'objectif essentiel des projets coloniaux, nous avons logiquement insisté sur les développements que connaît «la brousse » calédonienne aux dépens de la ville, Nouméa, qui, cependant, prend rapidement son essor ou encore de lieux spécifiques tels les chantiers miniers qui s'éparpillent sur les crêtes des montagnes en divers points. Le «rural» tient lieu de scène première, « l'urbain » et « l'industriel » jouent un rôle d'arrière-plan.

\section{Fondements et contradictions d'un projet de peuplement}

La Nouvelle-Calédonie occupe, dans l'histoire pénale française, une place singulière : une terre d'expérimentation sociale où il est possible, contrairement à la Guyane trop meurtrière, de pousser jusqu'à leur terme, les logiques de la loi de 1854 sur la transportation.

Ce texte rompt avec une longue tradition d'enfermement en imposant l'exil des criminels dans le but de protéger le corps social de leur néfaste présence. L'idée, cependant, dépasse la seule fonction répressive. Il s'agit, d'une part, de reproduire l'expérience menée par les Anglais, en Australie, à la fin du XVIII ${ }^{e}$ siècle et d'autre part de perpétuer dans un autre espace pénitentiaire, les buts moraux de "régénération» du condamné poursuivis jusqu'alors par les tenants de "la prison idéale ». A l'image des "convicts» anglais, les bagnards français seront expédiés en Nouvelle-Calédonie pour construire les infrastructures nécessaires au développement d'une colonie. Ecartés des influences pernicieuses du " vieux monde » et luttant contre une nature "sauvage », ils trouveront ainsi la « voie du retour au bien » et œuvreront pour leur propre avenir tout en stimulant l'arrivée d'une émigration "honnête", attirée par un pays déjà "outillé ». Cette dernière, en donnant l'exemple et en garantissant un équilibre dans la population qui ne saurait être exclusivement pénale, fait partie du processus de " régénération ». La France cherche à copier le modèle australien mais entend aussi le dépasser. Elle impose tout d'abord, aux condamnés, la règle de l'exil à vie et du doublage ${ }^{7}$ pour rendre leur retour impossible ou extrêmement difficile. En échange, elle promet de façon beaucoup plus systématique, la propriété $\mathrm{du}_{\mathrm{sol}}{ }^{8}$. Les valeurs sont là, bien françaises: la terre rédemptrice de tous les vices, favorisant la sérénité, l'amour du travail et de la famille. $C^{\prime}$ est aussi cette terre qui sera offerte aux migrants pauvres, issus de ces «classes laborieuses " potentiellement dangereuses.

En vertu de ces principes 22524 condamnés aux travaux forçés, 3928 déportés ${ }^{9}$ et 3796 relégués ${ }^{10}$ sont envoyés en Nouvelle-Calédonie entre 1864 et 1897. La logique de l'exil fonctionne à plein régime, la France expulsant des milliers d'hommes, généralement jeunes, condamnés à de lourdes peines principalement pour vol ou meurtre, issus dans leur grande majorité des couches les plus fragiles et instables de la société française, le prolétariat des campagnes et les petits métiers des villes. Les idéaux, en revanche, semblent rapidement se perdre dans les sables. Le premier décret d'application sur les 
concessions de terre accordées aux forçats ne sera publié qu'en 1878, soit 24 ans après la promulgation de la loi de $1854^{11}$. Dès 1887 , les orientations politiques changent pour privilégier l'installation du peuplement «libre et honorable». Au total, sur 22000 condamnés environ, 2717 seulement auront l'insigne honneur d'accéder au statut tant prisé de concessionnaire pénal, soit $10 \%$ de l'effectif global. Les $90 \%$ restants, forment ce qu'on appelle, en Nouvelle-Calédonie, les libérés. Relâchés à l'issue de leur peine avec pour seule richesse un pauvre baluchon, sommés de finir leur existence dans l'île en se débrouillant pour survivre, ces hommes symbolisent les contradictions flagrantes du système de la transportation. Ils en révèlent la vraie nature : un système qui sous couvert d'humanisme se contente de déplacer les problèmes en Nouvelle-Calédonie en concentrant là, une population qui ne cesse d'augmenter et à qui la colonie n'a rien à offriri $^{12}$.

L'émigration volontaire, difficile à chiffrer, représente un courant bien inférieur à celui de la transportation. La France, en ce domaine, se heurte à une incontournable réalité, celle d'un pays profondément enraciné dans ses terroirs et peu enclin à l'expatriation. S'il est possible d'expulser sur une vaste échelle les condamnés, il est beaucoup plus difficile de convaincre les Français de partir aux antipodes, s'installer dans une île de «bagnards et de sauvages ». La propagande du ministère des Colonies s'emploie à vanter les mérites "d'un pays sans hiver ", un climat doux et sain, une nature généreuse, des terres brunes et grasses. En accordant la gratuité du voyage et des concessions, il parvient à pousser dans l'aventure, des migrants qui, entre 1880 et 1890, offrent un profil social bien particulier présentant d'étonnantes similitudes avec celui des transportés. Les familles que nous avons étudiées, si elles n'ont pas «fauté », sont issues de ces mêmes couches instables et pauvres, rurales ou urbaines. Il s'agit d'une population, initialement mal intégrée dans son environnement, particulièrement touchée par l'exode rural précoce que connaît le $\mathrm{XIX}^{\mathrm{e}}$ siècle. Le départ aux colonies, dans un tel contexte, étroitement conditionné par les aides de l'État, peut s'analyser comme « un retour à la terre », une ultime tentative pour gagner un statut de possédant et échapper ainsi à la condition de prolétaire. Inscrite dans la continuité des mobilités internes françaises, l'émigration aux colonies s'interprète, au niveau individuel, comme une échappatoire par rapport au mouvement d'industrialisation, une quête d'identité rurale encore dominante mais déjà menacée ${ }^{13}$.

16 Faisant fi d'une conception sociale de la colonisation, la propagande cherche dans les dix dernières années du siècle à toucher des classes plus aisées possédant un capital de départ ${ }^{14}$; des candidats dynamiques et entreprenants qui sauront rapidement " mettre en valeur » un territoire désormais pensé comme « une petite France australe», propre et honnête, garante de l'influence de la nation dans le Pacifique ${ }^{15}$. Au nom de l'Empire et de la grandeur de la Patrie, quelques ingénieurs, docteurs, pharmaciens et autres notables viennent s'échouer dans les vallées calédoniennes. La tentative, cependant, tourne rapidement court. Brisant le mythe d'une terre de peuplement, la France renonce à ses idéaux, dès les années 1900, et abandonne le pays à son sort, relégant bagnards et colons au rang des chimères d'un autre siècle.

\section{Formes et logiques d'une société coloniale}

17 Fondamentalement structurée autour d'un projet de petite colonisation rurale, la Nouvelle-Calédonie coloniale s'inscrit dans une problématique foncière tout à fait 
spécifique. Contrairement à l'évolution que connaît l'Algérie, les autorités locales refusent là, de privatiser l'espace en son entier et ce contre l'avis du ministère des Colonies $^{16}$. L'enjeu essentiel, lié aux logiques mêmes d'implantations pénales ou libres, consiste à maintenir un contrôle rigoureux sur la répartition des terres. Plutôt que d'accorder la propriété privée aux Kanaks et autoriser ainsi les ventes directes entre indigènes et colons, au risque d'une dilapidation rapide des ressources foncières, les autorités locales s'en tiennent au principe du cantonnement. Celui-ci permet de définir précisément des espaces inaliénables réservés aux autochtones, le domaine public étant $a$ contrario dessiné pour être progressivement, attribué aux bagnards ou petits colons. Cette dynamique, s'appuyant sur une raison pratique, est aussi justifiée par des préjugés. Les Kanaks, considérés comme des êtres particulièrement "sauvages", sont censés vivre dans un communisme primitif qui exclut l'accès à la propriété privée, symbole même des civilisations "évoluées ». Les autorités coloniales se sentent d'autant plus légitimées à reléguer les indigènes dans des réserves qu'elles les pensent promis à une disparition inéluctable.

18 C'est dans ce contexte de mises en réserves des Kanaks, contexte extrêmement violent induisant un processus fondamental d'exclusion, que s'installent les colons libres ou pénaux. Les sites, libérés de leurs premiers habitants, donnent naissance à des villages tirés au cordeau construits sur le même modèle : concessions de quatre à cinq hectares s'alignant le long des rivières sur les riches terres alluviales. Si les bagnards ou migrants échappent à un affrontement direct avec les Kanaks, tous, en revanche, se heurtent aux difficiles réalités de la Nouvelle-Calédonie. Loin de l'image d'Epinal présentée par la propagande, le pays se révèle, au contraire, dans toute sa dureté. Les cyclones, les inondations, les sécheresses, les invasions de sauterelles annihilent régulièrement les efforts accomplis. L'absence de routes, l'isolement, le dépaysement, l'altérité radicale que présentent la nature et la société Kanak accentuent le découragement ou le sentiment d'insécurité. A l'aune de la «lutte coloniale», nombreux sont ceux qui échouent puis renoncent. Les centres pénaux témoignent d'une considérable instabilité, les condamnés se succédant sur des terres laissées à moitié en friche. L'alcoolisme, les vols et l'abandon révèlent toute la misère d'hommes souvent solitaires, placés sur un lopin de terre avec quelques effets, une pelle et une pioche. Les centres libres présentent aussi une histoire chaotique, caractérisée par un fort turn-over au sein des communautés fondatrices. L'aventure coloniale qui devait garantir une promotion inespérée, tourne, pour beaucoup, au désastre, en se soldant par un repli sur Nouméa ou par de pathétiques demandes de rapatriement. Ceux qui restent, s'accrochent, triomphent des difficultés, peuvent d'autant mieux mesurer le prix de leur réussite. Ils gagnent leurs lettres de noblesse en accédant au fameux statut de colon. S'ouvrent alors, pour les «libres » tout au moins, les portes du pouvoir : l'utilisation de la main-d'oeuvre, kanak ou javanaise ${ }^{17}$, prouvant par le fait même une position rêvée de patron ${ }^{18}$.

19 En dépit de sévères épreuves et de nombreux échecs, émerge en ce début de siècle, un monde calédonien ancré dans ses terroirs, profondément attaché à la terre et au pays ; monde qui, in fine, répond aux espoirs dont le projet colonial était porteur : l'image d'une France des campagnes transplantée dans le Pacifique. La société «blanche» de NouvelleCalédonie reste, cependant, fondamentalement éclatée. La brousse s'oppose à Nouméa, les colons aux libérés, les "libres" aux pénaux ». Chaque centre de colonisation, véritable lieu identitaire, constitue un univers social particulier et perpétue l'histoire de sa fondation, celle d'un itinéraire d'émigration spécifique. Les uns magnifient un passé de 
" pionniers » venus volontairement, les autres tentent de refouler les souvenirs du bagne, chacun se distinguant soigneusement du voisin. Ces clivages spatiaux et symboliques, effets d'héritage, ordonnent une société rigoureusement segmentée et se maintiennent sur le long terme, jusqu'à la Seconde Guerre mondiale tout au moins. Il faudra attendre les années 1960 pour que se comble peu à peu le fossé séparant les descendants des deux grandes branches de peuplement : « les libres » et « les pénaux ».

Dans un tel contexte, la conscience d'appartenir à un seul et même groupe, n'a de sens que par rapport à l'Autre, à savoir le Kanak dont il convient de se distinguer radicalement. C'est par cette distinction farouche et constamment réaffirmée que le monde «Blanc » trouve l'élément fondamental de son unité et finalement de son identité. Les frontières qui séparent les deux communautés sont, en Nouvelle-Calédonie, multiples. Elles sont tout d'abord spatiales car les réserves indigènes forment des mondes clos, autant « d'espaces privés » dans lesquels les colons ne s'aventurent guère. Elles sont aussi institutionnelles; la législation indigène interdisant tout déplacement hors des réserves sans autorisation. Elles sont enfin symboliques; la culture kanak constituant, aux yeux des «Blancs ", un univers mystérieux, dangereux et surtout "sauvage » dont il faut impérativement se défendre pour protéger sa propre appartenance au «monde civilisé ». Pour les colons de brousse en particulier, confrontés à un univers étrange et étranger, existe le risque d'une perte progressive de références provoquant un basculement dans un mode de vie entre-deux, «mi-civilisé », «mi-sauvage ». D'où l'extrême attention à maintenir une distance culturelle ferme avec tout ce que représente la société kanak.

La Nouvelle-Calédonie, en celà, offre un cas tout à fait original d'une ségrégation fondée sur un enjeu crucial de culture bien plus que sur des distinctions liées à la couleur de peau. Le métissage, en effet, fréquent dans les premières années de la colonisation et utilisé comme recours, du fait du déséquilibre du sex-ratio au sein de la population "blanche", ne produit pas de groupes intermédiaires tels que "les Demis" à Tahiti. Chacun, se définit en fonction de la société au sein de laquelle il a grandit. Un enfant métis, très foncé de peau, élevé hors de la tribu et donc hors de la réserve, par son père, colon « blanc » et sa mère mélanésienne, se considère et est considéré comme « Blanc ». Il tendra à se marier avec d'autres métis ou mieux encore avec une fille «blanche », issue d'une famille sans « tache ", exclusivement « blanche » et sera, dans ce cas, plus ou moins bien accepté selon sa réussite en tant que colon. En cas d'échec ou de mésentente, on saura, certes, lui rappeler ses origines kanaks, révélant ainsi un racisme de peau latent mais toujours présent. Son appartenance identitaire, cependant, ne pourra totalement être remise en cause. A l'inverse, un enfant au teint pâle, élevé en tribu par sa mère mélanésienne, et résidant donc dans une réserve, se rattache à un lignage, à un clan, à la coutume, à la culture kanak. Il se pense indubitablement Kanak et quoique, parfois, physiquement plus «blanc » que le colon du voisinage, entre, au début du siècle, dans la catégorie "Indigène». Ces subtilités montrent à quel point le racisme s'appuie en Nouvelle-Calédonie sur la culture bien plus que sur la nature ${ }^{19}$. Les formes spatiales, institutionnelles et symboliques se conjuguent, au fond, pour favoriser «le développement séparé des races ».

Sur fond d'ignorance, d'incompréhension et de peur, la Nouvelle-Calédonie contemporaine véhicule encore ce lourd héritage qui pèse sur les consciences et sur les réalités du pays. Long est le chemin à parcourir pour réduire les distances et les préjugés. 


\section{NOTES}

1. Plusieurs travaux ont été effectués sur l'histoire de la Nouvelle-Calédonie ; travaux généraux ou au contraire pointus, laissant dans l'ombre de nombreuses questions. Parmi les principaux, cf A Bensa, Nouvelle-Calédonie, un paradis dans la tourmente, Paris : Gallimard (Découvertes), 1990 ; A Saussol, L'Héritage. Essai sur le problème foncier mélanésien en Nouvelle-Calédonie, Publication de la Société des Océanistes, $\mathrm{n}^{\circ}$ 40, Paris. : Musée de l'Homme, 1979 et Colonisation et problème foncier en Nouvelle-Calédonie, Thèse de doctorat d'État, Université de Bordeaux III, 1985 ; J. Dauphiné., Les spoliations foncières en Nouvelle-Calédonie (1853-1913), Paris : L'Harmattan, 1989.

2. Contexte sur le terrain assez tendu ne permettant pas un va-et-vient entre les communautés sans susciter méfiance ou même rejet chez les colons, mes principaux interlocuteurs.

3. Ces termes renvoient à un débat propre aux historiens du Pacifique Sud; débat opposant les tenants d'une histoire globale de l'expansion européenne dans le Pacifique qui met en avant le rôle des Grandes Puissances et de leurs agents officiels ou tout au moins importants (explorateurs, missionnaires etc.) et les tenants d'une histoire insulaire privilégiant la situation spécifique de chaque île, l'étude de groupes coloniaux particuliers, la position des autochtones et les relations entre les uns et les autres.

4. Pour l'émigration libre, les registres d'État-civil ont permis de retrouver les lieux de naissance ainsi que la profession exercée avant le départ. Nous avons remonté les antécédents familiaux en interrogeant les archives des départements d'origine. Les états-civils ont aussi permis, pour l'émigration libre comme pour la colonisation pénale, de compléter les généalogies.

5. Il existe, une autre forme d'émigration volontaire qui pourrait être qualifiée de « spontanée ». Caractéristique de la période antérieure à la grande révolte Kanak de 1878, cette émigration concerne des individus arrivés par leurs propres moyens: aventuriers de tout poil, marins, mineurs, chercheurs d'or ou affairistes ainsi que des émigrants de toute origine provenant des colonies anglaises voisines. Ce courant migratoire recouvre des destins très divers dont seule une approche biographique pourrait rendre compte.

6. Pour reprendre la formule utilisée par Leroy-Beaulieu in De la colonisation chez les peuples modernes, Paris, 1874.

7. Les condamnés à 8 ans et plus sont exilés pour le restant de leur existence. Ceux qui sont condamnés à moins de 8 ans sont soumis à l'obligation de résidence pendant une période équivalente à la peine encourue. La condamnation minimale aux travaux forcés étant de 5 ans, tous les transportés sont sommés de passer au moins 10 ans en Nouvelle-Calédonie; temps plus que suffisant pour couper tout lien avec la Métropole, d'autant que pèse sur l'individu l'opprobre du bagne. Les convicts anglais, quant à eux, condamnés à l'exil pour 7 ou 14 ans avaient toujours le droit de rentrer en Grande-Bretagne, une fois leur peine achevée.

8. Le système de transportation, en Australie, ne se fixe pas comme objectif essentiel, l'octroi de terres aux ex-condamnés.

9. Les déportés, amnistiés par les lois de 1879 et 1881, regagneront, dans leur immense majorité, la métropole. S'ils ont joué un rôle essentiel dans la dénonciation du bagne calédonien, ils n'ont laissé localement qu'une faible empreinte. La déportation des communards en NouvelleCalédonie doit d'ailleurs s'analyser comme un événement contingent, lié aux imprévisibles soubresauts de l'évolution politique française. La IIIème République naissante l'intègre de façon tout à fait opportuniste au schéma global de la transportation.

10. Multi-récidivistes condamnés à l'exil en vertu de la loi du 27 mai 1885 qualifiée de «loi scélérate » par Robert Badinter dans son livre La Prison Républicaine, Fayard, 1992, p 165. Les 
relégués, représentants par excellence de la petite délinquance, subissent, en effet, en NouvelleCalédonie, une véritable condition de bagnards alors même qu'ils ont déjà effectué leur peine de prison, en France. Méprisés par tous (transportés y compris), beaucoup mèneront une existence misérable et très rares sont ceux qui obtiendront une concession de terre.

11. Les premiers villages pénaux s'ouvrent en 1869-1870, sur l'initiative du Gouverneur local. Ce n'est qu'en 1878 que la France s'engage concrètement pour soutenir et développer le projet de colonisation pénale.

12. Sur ce point cf I. Merle., 1992, "The trials and tribulations of the Emancipists: The consequences of penal colonisation in New Caledonia, 1864-1920 », in Robert Aldrich (ed), France, Oceania and Australia, Sydney University Press, p. 40-55.

13. I. Merle, "Genèse d'une identité coloniale. Histoire d'une émigration »organisée» vers la Nouvelle-Calédonie de la fin du XIX ${ }^{\mathrm{e}}$ siècle. La fondation des centres de Koné et Voh, 1880-1892 », Genèses, 13 (1993) 76-97.

14. Les migrants doivent désormais posséder 5000 francs-or au moment de leur départ.

15. La transportation vers la Nouvelle-Calédonie est suspendue en 1897 ; le pays étant alors voué à la seule colonisation "libre " conformément à une conception de peuplement offensive s'inquiétant des milliers d'Européens qui, dans le même temps, partent vers les États-Unis, l'Australie ou la Nouvelle-Zélande.

16. Le cantonnement est abandonné en Algérie en 1873 ; la loi Warnier imposant la propriété privée aux indigènes pour créer un domaine foncier soumis à la loi du marché. En quelques années de spéculations intenses, une grande majorité d'Algériens est expropriée au profit de colons qui se constituent d'immenses domaines. Cf. R. Ageron, Les Algériens Musulmans et la France, 1871-1919, PUF, 1968 ; P. Bourdieu et A. Sayad, Le Déracinement, Éditions de Minuit, 1964.

17. Travailleurs recrutés sous contrat pour servir en Nouvelle-Calédonie sur les propriétés des colons. Les Kanaks constituent une main-d'œuvre saisonnière mobilisée lors des récoltes de café ; la grande richesse agricole du pays. Les Tonkinois, recrutés dans les mêmes conditions que les Javanais, seront utilisés sur les mines.

18. L'accès à la main-d'œuvre est une étape essentielle de la promotion coloniale à laquelle les concessionnaire pénaux n'accèdent que tardivement. L'Administration Pénitentiaire exige, en effet, que les bagnards ou ex-bagnards travaillent par eux-mêmes, sans aide extérieure.

19. A Bensa, "Colonialisme, racisme et ethnologie en Nouvelle-Calédonie ", Ethonologie française, XVIII.2 (1988), p. 189-197. 